\title{
FATORES DE RISCO ASSOCIADOS A NEOPLASIA DE SISTEMA NERVOSO CENTRAL
}

\section{ARTIGO DE REVISÃO}

VASCONCELOS, Isys Holanda Albuquerque de ${ }^{1}$

PINHEIRO, Chrislaina Fernandes ${ }^{2}$

ROCHA, Andreza Carcará ${ }^{3}$

LEITE, Bruna Lívia Jorge ${ }^{4}$

CARNEIRO, Laura Sousa Dias ${ }^{5}$

BARROS, Lucas Soares Brandão ${ }^{6}$

VASCONCELOS, Marcela Karem de ${ }^{7}$

BARATELA, Maria Cecília ${ }^{8}$

MEDEIROS, Sabrina Rocha ${ }^{9}$

${ }^{1}$ Discente do curso de Medicina do Centro Universitário UNINTA, Sobral - CE.

2 Orientadora. Médica residente em Clínica Médica.

${ }^{3}$ Discente do curso de Medicina do Centro Universitário Unifacid (Unifacid).

4 Discente do curso de Medicina do Centro Universitário Maurício de Nassau (UNINASSAU).

5 Discente do curso de Medicina do Centro Universitário Maurício de Nassau (UNINASSAU).

${ }^{6}$ Discente do curso de Medicina da Universidade federal do Maranhão.

7 Discente do curso de Medicina do Centro Universitário Unifacid (Unifacid).

8 Discente do curso de Medicina da CeuniFametro.

${ }^{9}$ Discente do curso de Medicina da Universidade Federal do Espírito Santo (UFES). 
FREITAS, Vanessa de Oliveira ${ }^{10}$

VASCONCELOS, Isys Holanda Albuquerque de. Et al. Fatores de risco associados a neoplasia de sistema nervoso central. Revista Científica Multidisciplinar Núcleo do Conhecimento. Ano 06, Ed. 01, Vol. 02, pp. 89-104. Janeiro de 2021. ISSN: 24480959, Link de acesso: https://www.nucleodoconhecimento.com.br/saude/neoplasiade-sistema

\section{RESUMO}

Objetivo: Evidenciar os fatores de riscos associados a neoplasia de sistema nervoso central, a fim de melhor realizar rastreamentos. Método: É caracterizada por uma revisão integrativa, com métodos qualitativos e propriedades de aplicação, que visa determinar os fatores de risco relacionados aos tumores do sistema nervoso central. Executou-se a pesquisa acessando o banco de dados do PubMed. Para isso, utilizouse de descritores, contidos no MeSH (Medical Subject Headings), condizentes à temática da pesquisa e referentes aos termos: " risk factor", "association" , "astrocytoma" , "glioma", "oligodendroglioma" e "meningioma". Resultados: Foram analisados 20 estudos, dentre eles houve uma fração significativa de Caso-Controle, $75 \%(n=15)$. Do total de estudos explorados, 30\% $(n=6)$ foram sobre distúrbios metabólicos, 5\% $(n=1)$ abordaram hormonioterapias, 15\% $(n=3)$ analisaram fatores individuais e $25 \%(n=5)$ observaram relações com exposição ocupacional. Os demais $25 \%(n=5)$ foram estudos do tipo Coorte, nos quais 15\% $(n=3)$ abordaram síndromes metabólicas, e os outros $10 \%(n=2)$ tiveram a temática centrada em hormonioterapias e fatores individuais. Além disso, nota-se que a data de publicação desses artigos está entre os anos de 2015 e 2020. Conclusão: Os estudos avaliados, evidenciaram pontos como fatores individuais, exposição ocupacional, hormonioterapias e sinais de síndrome metabólicas como variáveis, que podem influenciar no risco para neoplasia do sistema nervoso central. Constatou se que, ferro, amianto, formaldeído demonstraram estar relacionados ao desenvolvimento de meningioma ou glioma. Já em relação a capacidade de resposta do organismo, mesmo de forma controversa

${ }^{10}$ Discente do curso de Medicina da Universidade Federal do Pará (UFPA). 
entre alguns autores, indivíduos que apresentam mais condições alérgicas, parecem estar protegidos dessa afecção, assim como pacientes com diabetes e que tomam medicação para o controle da mesma, foram associados a uma diminuição do risco de gioblastoma. As síndromes metabólicas e seus fatores de risco apresentaram-se como elementos relevantes no desenvolvimento dos tumores, enquanto o IMC e a hipertensão arterial são fatores que sofrem divergências para o risco de meningiomas. Via de regra, é fundamental investir no acompanhamento de pacientes acometidos por essas neoplasia, a fim de investigar fontes preveníveis.

Palavras-chave: Fatores de risco, neoplasia, sistema nervoso central.

\section{INTRODUÇÃO}

Os tumores primários do sistema nervoso central (SNC) caracterizam cerca de 2 a $3 \%$ de todos os tipos de neoplasias em adultos, e apresentam morbimortalidade elevada. É uma classe heterogênea de neoplasias, que acometem todos os intervalos de idade e têm potencial de acometimento tanto em medula e em qualquer ponto anatômico cerebral. São observadas como a segunda maior causa de morte no âmbito de doenças neurológicas, e em primeiro lugar estão os acidentes vasculares cerebrais. A incidência global de tumores cerebrais é cerca de 14,8/100.000 por ano, e metade tem classificação histológica benigna. (BERTOLUCCl et al, 2011).

Os tumores neurogliais representam cerca de $80 \%$ das neoplasias primárias, sendo derivados de astrócitos, oligodendrogliócitos e referidos em conjunto como gliomas. A Organização Mundial da Saúde (OMS) sugere uma classificação para esses tumores, são divididos em 4 graus (de grau I até grau IV). Segundo essa classificação, os gliomas são divididos em graus histológicos interligados conforme com o seu comportamento biológico, onde grau I e II são denominados de baixo grau e os de grau III e IV de alto grau. De acordo com uma pesquisa realizada pelo US Central Registry of Brain Tumors entre 2005 e 2009. Os registros totalizaram 311.202 registros. Meningioma é o tumor primário mais comum do sistema nervoso central (35,5\%), seguido pelo glioblastoma Tumor (15,8\%). As localizações mais comuns são as meninges $(35,2 \%)$ e a hipófise $(15,3 \%)$. 
Pode-se dizer, em geral, que o aparecimento dos tumores é de maneira esporádica, e não predomina causa específica. Apesar disso, alguns fatores são considerados de risco e foram reconhecidos. Os mais significativos são a história familiar e a exposição prévia à radiação ionizante. Entre outros pertinentes são: idade avançada, sexo masculino, infecção por HIV e ingestão de substâncias com nitrosamina. É importante pontuar que a extensão tumoral e a clínica do paciente são cruciais para um tratamento com obtenção de um prognóstico melhor. $\mathrm{O}$ tratamento tem como objetivo controlar a elevação da pressão intracraniana e o tumor. (HOFF et al, 2013). Portanto, é de suma relevância caracterizar os fatores de risco associados a neoplasias, afim de reduzir a mortalidade por essa causa.

O objetivo do estudo foi evidenciar os fatores de riscos associados a neoplasia de sistema nervoso central, a fim de melhor realizar rastreamentos.

\section{DESENVOLVIMENTO}

\subsection{METODOLOGIA}

É caracterizada por uma revisão integrativa, com métodos qualitativos e propriedades de aplicação, que visa determinar os fatores de risco relacionados aos tumores do sistema nervoso central.

Executou-se a pesquisa acessando o banco de dados do PubMed. Para isso, utilizouse de descritores, contidos no MeSH (Medical Subject Headings), condizentes à temática da pesquisa e referentes aos termos: " risk factor", "association" , "astrocytoma" , "glioma", "oligodendroglioma" e "meningioma" . Esses descritores foram usados em português, inglês e espanhol associados aos operadores booleanos "AND" e "OR" que forneceram a fórmula de pesquisa utilizada.

A busca realizada encontrou um total de 522 artigos na base de dados consultada, os quais foram selecionados por meio da leitura dos títulos e resumos. Fora utilizado como critérios de inclusão: publicações entre 2015 e 2020, de artigos que descreviam temas consistentes com os objetivos da pesquisa, que foram realizados em seres 
humanos, escritos em português, inglês e espanhol, e indexados na base de dado. Esses artigos estão todos disponíveis. Além disso, os critérios de exclusão descartaram artigos que não estavam disponíveis na íntegra e não atendiam ao objetivo da pesquisa. Após a aplicação destas especificações, obteve-se um total de 20 artigos, os quais foram posteriormente referidos.

Portanto, ressaltamos que o estudo não é prático, não havendo necessidade de submissão ao Comitê de Ética em Pesquisa (CEP).

Para apresentar os resultados desta revisão, pesquisa foi dividida por tópicos e organizada em uma tabela. Descrevendo as principais informações das amostras de artigos incluídas neste artigo, como nome do autor, ano de publicação, intervenção, tipo de pesquisa e os principais resultados encontrados em cada uma delas.

\subsection{RESULTADOS}

Tabela1: Fatores de risco associados a Neoplasia de sistema nervoso Central.

\begin{tabular}{|c|c|c|c|}
\hline Autor, ano & $\begin{array}{l}\text { Tipo de } \\
\text { estudo }\end{array}$ & Objetivos & Resultados \\
\hline $\begin{array}{l}\text { R Rogers et } \\
\text { al. (2020) }\end{array}$ & $\begin{array}{l}\text { Estudo de } \\
\text { coorte } \\
\text { retrospectivo }\end{array}$ & $\begin{array}{l}\text { Investigar } \\
\text { Síndrome } \\
\text { metabolica como } \\
\text { fator de risco e } \\
\text { fator que afeta a } \\
\text { sobrevida no } \\
\text { glioblastoma. }\end{array}$ & $\begin{array}{l}\text { Identificou-se síndrome } \\
\text { metabólica em uma frequência } \\
\text { ligeiramente maior em } \\
\text { pacientes diagnosticados com } \\
\text { glioblastoma em comparação } \\
\text { com a população geral. E } \\
\text { também está associada a um } \\
\text { pior prognóstico geral. }\end{array}$ \\
\hline $\begin{array}{l}\text { Wiedmann } \\
\text { MKH et al. } \\
\text { (2017) }\end{array}$ & $\begin{array}{l}\text { Estudo de } \\
\text { coorte } \\
\text { prospectivo }\end{array}$ & $\begin{array}{l}\text { Avaliar se a } \\
\text { gordura corporal ou } \\
\text { a altura corporal } \\
\text { estão associadas } \\
\text { ao risco de }\end{array}$ & $\begin{array}{l}\text { A obesidade não foi fator de } \\
\text { risco para meningioma e } \\
\text { glioma, mas foi } \\
\text { significativamente associada } \\
\text { ao risco de adenoma }\end{array}$ \\
\hline
\end{tabular}




\begin{tabular}{|l|l|l|l|}
\hline & & $\begin{array}{l}\text { meningioma, } \\
\text { glioma, adenoma } \\
\text { hipofisário (PA) ou } \\
\text { tumor da bainha } \\
\text { nervosa (NST) }\end{array}$ & $\begin{array}{l}\text { hipofisário. Para NSTs } \\
\text { intracranianos o risco de tumor } \\
\text { foi reduzido. A altura foi fator de }\end{array}$ \\
risco para os quatro subgrupos \\
de tumor.
\end{tabular}




\begin{tabular}{|c|c|c|c|}
\hline & & & $\begin{array}{l}\text { doença inflamatória intestinal, } \\
\text { doenças da glândula tireóide, } \\
\text { vasculites, alergias. Não houve } \\
\text { risco ligado ao uso de } \\
\text { corticosteroides, } \\
\text { corticosteroides sistêmicos ou } \\
\text { de medicamento } \\
\text { imunossupressor. }\end{array}$ \\
\hline $\begin{array}{l}\text { Seliger C et } \\
\text { al. (2015) }\end{array}$ & $\begin{array}{l}\text { Estudo de } \\
\text { caso- } \\
\text { controle }\end{array}$ & $\begin{array}{l}\text { Examinar o status } \\
\text { da diabetes, } \\
\text { duração da } \\
\text { diabetes, controle } \\
\text { glicêmico e uso de } \\
\text { agentes } \\
\text { antidiabéticos } \\
\text { comuns em relação } \\
\text { ao risco de glioma }\end{array}$ & $\begin{array}{l}\text { Ter diabetes foi associado a } \\
\text { uma diminuição do risco de } \\
\text { glioma, particularmente } \\
\text { glioblastoma, principalmente } \\
\text { em homens com diabétes de } \\
\text { longa duração. Nenhuma } \\
\text { associação significativa com } \\
\text { glioma foi encontrada para o } \\
\text { uso de metformina } \\
\text { sulfonilureias ou insulina. }\end{array}$ \\
\hline $\begin{array}{l}\text { Seliger C et } \\
\text { al. (2017) }\end{array}$ & $\begin{array}{l}\text { Estudo de } \\
\text { caso- } \\
\text { controle }\end{array}$ & $\begin{array}{l}\text { Analisar o status do } \\
\text { diabetes, curso do } \\
\text { diabetes, controle } \\
\text { do açúcar no } \\
\text { sangue e uso de } \\
\text { metformina, } \\
\text { sulfonilureias e } \\
\text { insulina em relação } \\
\text { ao risco de } \\
\text { meningioma }\end{array}$ & $\begin{array}{l}\text { Houve a sugestão de uma } \\
\text { associação inversa com } \\
\text { meningioma e a duração da } \\
\text { diabetes, principalmente nas } \\
\text { mulheres. Para a metformina, } \\
\text { houve uma relação positiva } \\
\text { sugestiva, principalmente após } \\
\text { o pareamento da duração do } \\
\text { diabetes e do nível de HbA1c. } \\
\text { As sulfonilureias não } \\
\text { mostraram associação clara. } \\
\text { Para a insulina, houve } \\
\text { sugestão de relação inversa. }\end{array}$ \\
\hline
\end{tabular}




\begin{tabular}{|c|c|c|c|}
\hline $\begin{array}{l}\text { Seliger C et } \\
\text { al. (2016) }\end{array}$ & $\begin{array}{l}\text { Estudo de } \\
\text { caso- } \\
\text { controle }\end{array}$ & $\begin{array}{ll}\text { Avaliar todos os } & \text { da } \\
\text { componentes } & \text { de } \\
\text { DM em relação ao } & \text { disco } \\
\text { meningioma. }\end{array}$ & $\begin{array}{l}\text { O IMC está positivamente } \\
\text { correlacionado com } \\
\text { meningioma. A hipertensão } \\
\text { arterial também foi associada a } \\
\text { um risco aumentado de } \\
\text { meningioma. Em comparação, } \\
\text { lipoproteína de alta densidade, } \\
\text { triglicerídeos, glicose sérica em } \\
\text { jejum e uso de inibidores da } \\
\text { ECA, inibidores de AT-II, beta- } \\
\text { bloqueadores, diuréticos, } \\
\text { antagonistas de cálcio, nitratos } \\
\text { ou estatinas não foram } \\
\text { associados ao risco de } \\
\text { meningioma. }\end{array}$ \\
\hline $\begin{array}{l}\text { Sadetzki } S \\
\text { et al.(2016) }\end{array}$ & $\begin{array}{l}\text { Estudo de } \\
\text { caso- } \\
\text { controle }\end{array}$ & $\begin{array}{l}\text { Examinar as } \\
\text { associações entre } \\
\text { a exposição } \\
\text { ocupacional a } \\
\text { metais e o risco de } \\
\text { meningioma }\end{array}$ & $\begin{array}{l}\text { A exposição ao Fe foi } \\
\text { significativa estatisticamente } \\
\text { para o desenvolvimento de } \\
\text { meningioma, principalmente } \\
\text { entre as mulheres. }\end{array}$ \\
\hline $\begin{array}{l}\text { Turner MC } \\
\text { et al.(2017) }\end{array}$ & $\begin{array}{l}\text { Estudo de } \\
\text { caso- } \\
\text { controle }\end{array}$ & $\begin{array}{l}\text { Avaliação da } \\
\text { exposição } \\
\text { produtos } \\
\text { e químicos } \\
\text { magnéticos } \\
\text { extrema de } \\
\text { frequência } \\
\text { etiologia do tumor } \\
\text { cerebral. }\end{array}$ & $\begin{array}{l}\text { Não houve evidência } \\
\text { significativa entre ELF e as } \\
\text { exposições químicas para o } \\
\text { risco de glioma e meningioma. }\end{array}$ \\
\hline
\end{tabular}




\begin{tabular}{|c|c|c|c|}
\hline $\begin{array}{l}\text { McElvenny } \\
\text { DM et } \\
\text { al.(2018) }\end{array}$ & $\begin{array}{l}\text { Estudo de } \\
\text { caso- } \\
\text { controle }\end{array}$ & $\begin{array}{l}\text { Estudar se o risco } \\
\text { de meningioma } \\
\text { está associado a } \\
\text { várias exposições } \\
\text { ocupacionais, } \\
\text { incluindo produtos } \\
\text { de combustão } \\
\text { selecionados, pós } \\
\text { e outros agentes } \\
\text { químicos }\end{array}$ & $\begin{array}{l}\text { Foi } \\
\text { observada estatisticamente } \\
\text { significativa a relação com a } \\
\text { exposição cumulativa e a } \\
\text { duração da exposição. Nas } \\
\text { mulheres houve tendência } \\
\text { significativa dessa relação com } \\
\text { o amianto e excessos nas } \\
\text { categorias de exposição mais } \\
\text { altas para o formaldeído. }\end{array}$ \\
\hline $\begin{array}{l}\text { Benke G et } \\
\text { al. (2017) }\end{array}$ & $\begin{array}{l}\text { Estudo de } \\
\text { caso- } \\
\text { controle }\end{array}$ & $\begin{array}{l}\text { Examinar } r \text { a } \\
\text { associação entre } \\
\text { uma gama de } \\
\text { solventes e risco } \\
\text { de glioma. }\end{array}$ & $\begin{array}{l}\text { Não houve associação entre a } \\
\text { exposição a qualquer solvente } \\
\text { e o risco de glioma geral. }\end{array}$ \\
\hline $\begin{array}{l}\text { Parent ME et } \\
\text { al. (2017) }\end{array}$ & $\begin{array}{l}\text { Estudo de } \\
\text { caso- } \\
\text { controle }\end{array}$ & $\begin{array}{l}\text { Estudar o risco de } \\
\text { glioma e a } \\
\text { exposição a cinco } \\
\text { metais - chumbo, } \\
\text { cádmio, níquel, } \\
\text { cromo e ferro- e } \\
\text { fumos de soldagem }\end{array}$ & $\begin{array}{l}\text { Houve prevalência pouco } \\
\text { significativa dos casos em } \\
\text { relação aos controles quando } \\
\text { exposição a vários metais e } \\
\text { fumos de soldagem. }\end{array}$ \\
\hline $\begin{array}{l}\text { Ben-Zion } \\
\text { Berliner et } \\
\text { al. (2020) }\end{array}$ & $\begin{array}{l}\text { Estudo de } \\
\text { corte }\end{array}$ & $\begin{array}{l}\text { Investigar os } \\
\text { diferentes fatores } \\
\text { de riscos no } \\
\text { desenvolvimento } \\
\text { de meningioma em } \\
\text { adolescentes e } \\
\text { jovens adultos. }\end{array}$ & $\begin{array}{l}\text { Identificou-se miningioma em } \\
\text { uma frequência maior em } \\
\text { pacientes do sexo feminino. } \\
\text { Além disso, em pacientes do } \\
\text { sexo masculino, a altura se } \\
\text { mostrou um fator significativo. }\end{array}$ \\
\hline
\end{tabular}




\begin{tabular}{|c|c|c|c|}
\hline $\begin{array}{l}\text { Zhang D. et } \\
\text { al (2016) }\end{array}$ & $\begin{array}{l}\text { Caso- } \\
\text { Controle e } \\
\text { Coorte }\end{array}$ & $\begin{array}{lr}\text { Investigar } & \text { os } \\
\text { dados } & \text { que } \\
\text { relacionam o } & \text { IMC } \\
\text { (Índice de } & \text { Massa } \\
\text { Corpórea) com } & \text { o } \\
\text { risco } & \text { do } \\
\text { desenvolvimento } \\
\text { tumores cerebrais, } \\
\text { especialmente os } \\
\text { gliomas } \\
\text { meningiomas. }\end{array}$ & $\begin{array}{l}\text { Foi estabelecido que o excesso } \\
\text { de peso está associado a um } \\
\text { risco aumentado de tumores } \\
\text { cerebrais e meningiomas (em } \\
\text { vez de gliomas). }\end{array}$ \\
\hline $\begin{array}{l}\text { S Benson et } \\
\text { al. (2015) }\end{array}$ & Coorte & $\begin{array}{lr}\text { Investigar } & \text { os } \\
\text { dados r que } & \text { qacionam } \\
\text { rela } \\
\text { a rerapia } \\
\text { Hormonal ra na } \\
\text { Menopausa com } \\
0 \quad \text { risco do } \\
\text { desenvolvimento } \\
\text { de tumores no } \\
\text { Sistema Nervoso } \\
\text { Central. }\end{array}$ & $\begin{array}{l}\text { Identificou-se o aumento do } \\
\text { risco de tumores } \\
\text { cerebrais,glioma } \\
\text { meningioma, em pacientes } \\
\text { que fizeram o somente o uso } \\
\text { do estrogênio, mas não dos } \\
\text { usuários de progestina e } \\
\text { estrogênio. }\end{array}$ \\
\hline $\begin{array}{l}\text { K H } \\
\text { WIEDMANN } \\
\text { et al (2017) }\end{array}$ & Coorte & $\begin{array}{l}\text { Investigar o IMC } \\
\text { como fator de risco } \\
\text { para diferentes } \\
\text { subgrupos } \\
\text { de gliomas }\end{array}$ & $\begin{array}{l}\text { Identificou-se que o excesso } \\
\text { de peso e a obesidade não } \\
\text { estão associados a risco de } \\
\text { nenhum subgrupo de glioma }\end{array}$ \\
\hline $\begin{array}{l}\text { TAKAHASHI } \\
\text { et al (2019) }\end{array}$ & $\begin{array}{l}\text { Caso- } \\
\text { Controle }\end{array}$ & $\begin{array}{l}\text { Investigar os dados } \\
\text { das variantes } \\
\text { genéticas } \\
\text { associadas a }\end{array}$ & $\begin{array}{l}\text { Identificou-se que a análise } \\
\text { deu suporte para a associação } \\
\text { entre obesidade e o risco } \\
\text { acentuado de meningioma. }\end{array}$ \\
\hline
\end{tabular}




\begin{tabular}{|c|c|c|c|}
\hline & & $\begin{array}{l}\text { obesidade e sua } \\
\text { relação com o risco } \\
\text { de meningioma, } \\
\text { utilizando } \\
\text { Randomização } \\
\text { Mendeliana. }\end{array}$ & \\
\hline $\begin{array}{l}\text { SMUSKENS } \\
\text { et al (2019) }\end{array}$ & $\begin{array}{l}\text { Caso- } \\
\text { Controle }\end{array}$ & $\begin{array}{l}\text { Investigar o IMC, } \\
\text { as comorbidades e } \\
\text { os fatores } \\
\text { hormonais em } \\
\text { relação ao risco de } \\
\text { meningioma em } \\
\text { uma população } \\
\text { etinicamente } \\
\text { diversa. }\end{array}$ & $\begin{array}{l}\text { Identificou-se que a } \\
\text { associação de Obesidade com } \\
\text { meningioma foi observada } \\
\text { em Nipo-americanos, Nipo- } \\
\text { americanos hipertensos e } \\
\text { nativos havaianos. }\end{array}$ \\
\hline $\begin{array}{l}\text { DISNEY - } \\
\text { HOGG et al } \\
(2018)\end{array}$ & $\begin{array}{l}\text { Caso- } \\
\text { Controle }\end{array}$ & $\begin{array}{l}\text { Investigar a relação } \\
\text { inversa entre } \\
\text { alergias e o risco } \\
\text { de gliomas, } \\
\text { utilizando } \\
\text { Randomização } \\
\text { Mendeliana }\end{array}$ & $\begin{array}{l}\text { Identificou-se que a pesquisa } \\
\text { não encontrou evidências } \\
\text { fortes o suficiente para achar } \\
\text { relação entre dermatite atópica } \\
\text { e o risco de desenvolver um } \\
\text { glioma }\end{array}$ \\
\hline $\begin{array}{l}\text { POUCHIEU } \\
\text { et al (2017) }\end{array}$ & $\begin{array}{l}\text { Caso } \\
\text { controle }\end{array}$ & $\begin{array}{l}\text { Analisar a relação } \\
\text { entre o histórico de } \\
\text { alergias e o risco } \\
\text { de glioma e } \\
\text { meningioma,, } \\
\text { tendo em vista as } \\
\text { condições acerca } \\
\text { dos estilos de vida } \\
\text { em potencial, }\end{array}$ & $\begin{array}{l}\text { Foi examinado a associação } \\
\text { entre históricos de alergia e } \\
\text { risco de glioma e meningioma } \\
\text { em adultos, de forma que não } \\
\text { houve relação entre } \\
\text { meningioma e às alergia, ao } \\
\text { passo que houve associação } \\
\text { inversa entre condições } \\
\text { alérgicas e risco de glioma. }\end{array}$ \\
\hline
\end{tabular}




fatores de risco
ocupacionais e
ambientais.

Fonte: Próprio Autor.

Foram analisados 20 estudos, dentre eles houve uma fração significativa de CasoControle, $75 \%(n=15)$. Do total de estudos explorados, 30\% $(n=6)$ foram sobre distúrbios metabólicos, $5 \%(n=1)$ abordaram hormonioterapias, 15\% $(n=3)$ analisaram fatores individuais e $25 \%(n=5)$ observaram relações com exposição ocupacional. Os demais $25 \%(n=5)$ foram estudos do tipo Coorte, nos quais $15 \%(n=3)$ abordaram síndromes metabólicas, e os outros $10 \%(n=2)$ tiveram a temática centrada em hormonioterapias e fatores individuais. Além disso, nota-se que a data de publicação desses artigos está entre os anos de 2015 e 2020.

Acrescenta-se que, 9 artigos (45\%) que tiveram como tema as Síndromes metabólicas, relataram associações entre Obesidade e Diabetes às Neoplasias do sistema nervoso central. Tratando-se de relação com fatores individuais investigou-se sexo, histórico de alergias, tabagismo e IMC, $n=4(20 \%)$. Os 2 artigos (10\%) que abordaram hormonioterapias, relacionaram os hormônios, como estrogênio, aos riscos dessas neoplasias. Por fim, 5 estudos $(25 \%)$ relacionaram a exposição ocupacional às neoplasias, de forma que houve associação à exposição ao Ferro com o desenvolvimento de meningioma.

\subsubsection{EXPOSIÇÕES OCUPACIONAIS}

Em relação às exposições ocupacionais, há representação de $25 \%(n=5)$ do total dos artigos, além da utilização em 100\% $(n=5)$ do estudo do tipo Caso-Controle. Nessa perspectiva, foram analisados os ricos do desenvolvimento de Gliomas, de meningiomas e de tumores cerebrais associados às exposições aos metais como chumbo, cadmio, níquel, cromo e ferro. Além de riscos devido aos produtos químicos, aos campos magnéticos de extrema baixa frequência aos produtos de combustão e a outros agentes químicos. Dessa forma, constatou-se que exposição ao Ferro por 
mulheres foi um fator significativo para o desenvolvimento de meningiomas, de acordo estudos de (SADETZKI, 2016). Da mesma forma que houve relação com o amianto e às altas exposições ao formaldeído (MCELVENNY, 2018). Em contrapartida, houve prevalência pouco significativa à exposição aos outros tipos de metais (PARENT, 2017) e aos campos magnéticos de extrema baixa frequência no desenvolvimento de tumores (TURNER, 2017).

\subsubsection{SÍNDROMES METABÓLICAS}

Cerca de 47,36\% ( $n=9)$ dos trabalhos abordavam o impacto das síndromes metabólicas, segundo uma coorte prospectiva Rogers et al. (2020), elas foram observadas em uma frequência um pouco maior em pacientes diagnosticados com glioblastoma, e dessa forma também estavam correlacionadas a um pior prognóstico.

O estudo (SELIGER et al., 2015) obteve que para os pacientes diabéticos o risco para glioma do tipo glioblastoma foi reduzido, quando em homens com diabetes de longa duração. Já as mulheres diabéticas de longa duração apresentaram risco inverso para meningioma, o uso de insulina também foi sugestivamente inverso, no caso-controle (SELIGER et al., 2017). Houve sugestão de relação positiva desse mesmo tumor com metformina, IMC elevado e HAS (SELIGER et al., 2016). Em três trabalhos mostrouse acentuada a associação da obesidade e o risco para meningioma, principalmente em Nipo-americanos, Nipo-americanos com HAS e nativos havaianos, mas não mostrou significância para nenhum subgrupo de glioma. Já em um único trabalho (WIEDMANN et al., 2017) apresentou-se a obesidade como não fator de risco para meningioma e glioma, sendo mais significativo para adenoma hipofisário, bem como risco reduzido para NSTs intracranianos, nesse mesmo estudo a altura estava associada ao risco dos quatro subgrupos de tumores apresentados.

\subsubsection{FATORES INDIVIDUAIS}

Os fatores individuais foram abordados em $21,05 \%(n=4)$ dos trabalhos, mostrando que o risco para glioma era reduzido quando o indivíduo apresentava baixo IMC, insuficiência cardíaca, IC congestiva no sexo masculino e infarto do miocárdio prévio, 
e foi descartado esse risco associado a qualquer doença auto-imune (ANSSAR et al., 2019), esse caso-controle buscou analisar justamente essa última associação no aumento da vigilância imunológica. Ao investigar os fatores de risco do meningioma em adolescentes e jovens adultos, um estudo de coorte (BEN-ZION et al., 2010) apresentou o meningioma como sendo mais frequente no sexo feminino e a altura como fator de risco para o sexo masculino. Já em relação aos fatores alérgicos, um caso-controle identificou na sua investigação para o risco de glioma que existem evidências fortes para se relacionar a dermatite atópica e risco para glioma (DISNEYHOGG et al., 2018). Em outro caso controle, que também analisou o histórico médico de alergias em adultos, não se apresentou relação entre meningioma e as alergias, mas houve relação inversa delas como risco de glioma (POUCHIEU et al., 2017).

\subsubsection{HORMONIOTERAPIA}

Em 10,52\% ( $n=2)$ dos estudos investigou-se a terapia hormonal e o risco de tumores do SNC. Em um dos estudos os riscos relativos para a HT somente com estrogênio, foi maior que para HT com estrogênio e progesterona associados, em se tratando de todo os tumores do SNC, e em comparação dos usuários de HT com os nunca usuários o risco relativo também foi elevado (BENSON et al., 2014). Já no outro estudo, uma coorte (BENSON et al., 2015), o risco aumentado para os tumores do SNC, glioma e meningioma foi devido as pacientes que fizeram reposição hormonal na menopausa apenas com estrogênio, e também o risco não se apresentou para as que fizeram uso de estrogênio-proegestina.

\subsection{DISCUSSÃO}

\subsubsection{FATORES INDIVIDUAIS}

Valendo-se dos resultados de Disney-Hogg et al. (2018), os quais não identificaram evidências para sugerir a relação entre dermatite atópica e o risco de desenvolver glioma, e dos resultados de Pochieu et al. (2017), não encontraram relação entre meningioma e alergias, mas destacou que existe uma correlação negativa entre condições alérgicas e risco de glioma. A literatura sugere que pacientes com histórico 
de alergias podem ter riscos alterados para neoplasias malignas devido à desregulação da função do sistema imunológico tanto para o lado de risco: devido à presença dos processos inflamatórios, tornando o organismo mais susceptível à doença; quanto para o lado de proteção: com uma imunidade otimizada, a identificação e eliminação das células tumorais ocorre mais facilmente (JO et al., 2018).

$\mathrm{Na}$ literatura, há crescente concordância para a relação inversa entre condições alérgicas e risco de gliomas. Uma das possíveis explicações para isso seria que o aumento da atividade e vigilância imunológica seria capaz de diminuir essa associação (MICHAUD, 2019). No estudo de Lino et al. (2007) observou-se forte associação inversa entre incidência de gliomas e presença de alergias. No entanto, corroborando com nossos achados, eles também não encontraram relação entre processos alérgicos e desenvolvimento de meningiomas. Mais trabalhos precisam ser realizados para elucidar melhor a relação entre a fisiopatologia das condições alérgicas e o desenvolvimento de tumores em SNC.

Em relação aos nossos achados sobre a falta de associação entre gliomas e quaisquer doenças autoimunes, Brenner, et al. 2002 observaram uma relação inversa entre histórico médico de doenças autoimunes e meningiomas, embora não se tenha esclarecido qual seria a gênese dessa relação no tocante a função dos fatores de imunidade nos tumores cerebrais. Sendo assim, é necessário também, que mais trabalhos sejam realizados para que possamos compreender sobre a existência ou não desse tipo de associação.

\subsubsection{HORMONIOTERAPIA}

No que tange à hormonioterapia, encontramos associação entre mulheres que fazem reposição hormonal com estrogênio e o maior desenvolvimento de tumores em SNC. Entretanto, na literatura não encontramos trabalhos que testem essa associação, embora existam estudos que avaliem essa relação em neoplasias de outros sítios anatômicos. Por isso, novas pesquisas devem ser elaboradas para testar a hipótese de a terapia hormonal ser ou não um fator de risco para neoplasias de SNC. 


\subsubsection{EXPOSIÇÃO OCUPACIONAL}

Em ambientes ocupacionais, podem ser encontrados diferentes agentes cancerígenos, tais como: amianto, sílica, solventes aromáticos, metais pesados, radiação ionizante e agrotóxicos. Quando o trabalhador é exposto a alguns desses agentes e ainda a outras condições de risco comprovadamente cancerígenas, como: tabagismo, alcoolismo exagerado e dieta rica em gordura trans, a exposição se torna potencializada para a ocorrência de neoplasias malignas (HOFF et al., 2013).

Em relação à exposição a metais pesados que comprovadamente causam alterações no sistema nervoso central, temos o chumbo, encontrado em tintas, soldas e baterias. Ele consegue reagir com proteínas levando a alteração das suas funções e podendo originar encefalopatia saturnínica quando presente em SNC; o mercúrio é outro metal pesado encontrado em mineração e defensivos agrícolas que também pode levar à distúrbios no SNC, caracteristicamente, a síndrome neurastênicano (FILHO et al., 2018). McElven et al. (2018) identificaram que metais como o ferro e amianto tiveram maiores associações com o risco de desenvolver meningiomas; já no estudo de Parent et al. (2017) a prevalência foi pouco significativa dos casos em relação aos controles quando à exposição a vários metais e fumos de soldagem para o desenvolvimento de gliomas. Por isso, sabendo da possibilidade que os metais pesados têm em produzir modificações no SNC, não devemos excluí-los em novas pesquisas como possíveis fatores de risco para o desenvolvimento de neoplasias nesse sítio.

O estudo de Turner et al. (2017) não demonstrou evidência significativa entre campos magnéticos de baixa frequência para o risco de glioma e meningioma. Segundo Filho et al. (2018), não foi evidenciado na literatura aumento de risco para gliomas e meningiomas em indivíduos expostos à micro-ondas e campos eletromagnéticos em redes de alta tensão (FILHO et al., 2018).

Em mulheres, foi demonstrado no estudo de McElvenny et al. (2020), que a exposição ao formaldeído constituiu risco aumentado para meningiomas. Entretanto, nenhuma relação entre essa substância química e maior risco para o desenvolvimento de 
gliomas foi observado no estudo de Lacourt et al. (2018). Isso nos leva à questão de se é razoável testar relações entre fatores ocupacionais sabendo que eles estão associados a fatores individuais, como condição social, hábitos alimentares e estilo de vida, além da exposição a outros agentes físicos e químicos fora do ambiente de trabalho, uma vez que esses outros fatores também podem estar atuando para o maior risco de neoplasias em SNC. (HOFF et al., 2013).

\subsubsection{ASSOCIAÇÃO DA SÍNDROME METABÓLICA COM O GIOBLASTOMA}

De acordo com os estudos, uma grande parte dos indivíduos que possuem gioblastoma apresentam também Síndrome Metabólica (SN) como fator de risco, a qual sua sobrevida seja afetada. Em geral, a SM é identificada como fator de risco para o desenvolvimento de vários cânceres sistêmicos, mas sua frequência entre pacientes com glioblastoma e sua associação com desfechos clínicos estão em constante pesquisa. Com isso, tanto a síndrome metabólica quanto o desenvolvimento de glioblastoma compartilham fatores de risco em comum, como a idade, obesidade, a hipertensão arterial e a hiperglicemia. Ainda, de acordo com o estudo realizado por (ABESO et al., 2009), pacientes com gliomas apresentam sobrepeso e aumento da massa gorda, principalmente na região abdominal, que corrobora com o agravo dessas comorbidades.

\subsubsection{IMC ASSOCIADO AO MENINGIOMA}

De acordo com o estudo vigente, os índices antropométricos (altura, peso, IMC) possuem uma ligação direta para o risco de desenvolvimento de meningioma. O IMC e a hipertensão arterial foram associados a um risco aumentado de meningioma. Corroborando com essa ideia, Zhang et al. (2016), a adiposidade está relacionada ao risco aumentado para meningioma, mas não está associada ao risco de glioma. Com base em um conjunto limitado de evidências, a atividade física está relacionada à diminuição do risco de meningioma, mas mostra pouca associação com o risco de glioma. 
Além disso, existe um confronto de ideias em relação aos autores, pois Wiedmann et al. (2017) relata que o sobrepeso ou a obesidade não confirmam esses dois como fatores de risco para meningioma. Além disso, o sobrepeso e a obesidade podem ser bastante confiantemente excluídos como fatores de risco para glioma. Em suma, vale destacar o incremento de mais pesquisas nesse campo para que os dois pensamentos se alinhem em uma só direção.

\subsubsection{RELAÇÃO ENTRE DIABETES E GLIOMAS}

De acordo com os estudos, pacientes com diabetes e que tomam medicação para o controle da mesma foram associados a uma diminuição do risco de glioma, particularmente gioblastoma. Para corroborar com tal fato Schwartzbaum et al. (2017), sugeriu que tumores pré-clínicos podem consumir muita glicose no sangue, o que levaria a uma redução significativa do risco de glioma em pacientes diabéticos ou a um aumento nos níveis de glicose no sangue. Eles citaram um fenômeno chamado efeito Warburg pelo qual as células cancerígenas, em geral, consomem mais glicose do que células não proliferadoras. Os autores explicam que, se esse efeito existir, a correlação negativa entre os níveis do tumor e o açúcar no sangue será mais forte perto do momento do diagnóstico do glioma. No entanto, tal fato ainda deve ser muito mais pesquisado para obter dados mais concretos diante do exposto.

A maioria dos pacientes com níveis elevados de açúcar no sangue usa metformina como medicamento de controle, pois de acordo com Pollack et al. (2012), A metformina quase não tem efeitos colaterais e é barata. Independentemente do plano de tratamento do tumor, houve benefícios. Além disso, os resultados apresentados indicam que pode ser usado como uma terapia adjuvante para melhorar ainda mais a sobrevida, mesmo em pacientes recebendo tratamento padrão, a hipótese de que a metformina tem atividade anticâncer é suportada. A partir deste estudo, não podemos concluir se 0 efeito deste medicamento pode ser observado em populações não diabéticas e se os resultados são afetados pela diferença potencial entre pacientes tratados com metformina e pacientes tratados com insulina / sulfonilureia, ou se há apenas atividade anticâncer em pacientes com diabetes ou outras anormalidades metabólicas. 


\section{CONSIDERAÇÕES FINAIS}

Os estudos avaliados, evidenciaram que pontos como fatores individuais, exposição ocupacional, hormonioterapias e sinais de síndrome metabólicas como variáveis, podem influenciar no risco para neoplasia do sistema nervoso central. Constatou se que, ferro, amianto, formaldeído mostraram-se relevantes no desenvolvimento de meningiomas ou gliomas. Já em relação a capacidade de resposta do organismo, mesmo de forma controversa entre alguns autores, indivíduos que apresentam mais condições alérgicas, parecem estar protegidos dessa afecção, assim como pacientes com diabetes e que tomam medicação para o controle da mesma, foram associados a uma diminuição do risco de gioblastoma. As síndromes metabólicas e seus fatores de risco apresentaram-se como elementos relevantes no desenvolvimento dos tumores, enquanto o IMC e a hipertensão arterial são fatores que sofrem divergências para o risco de meningiomas. Via de regra, é fundamental investir no acompanhamento de pacientes acometidos por essas neoplasia, a fim de investigar fontes preveníveis.

\section{REFERÊNCIAS}

ABESO, Associação Brasileira para o Estudo da Obesidade e da Síndrome Metabólica. Diretrizes Brasileiras de Obesidade. ABESO $3^{\circ}$ Edição , [s. I.], p. 1-85, 3 ago. 2009. Disponível em: https://www.passeidireto.com/arquivo/48198180/diretrizesbrasileiras-de-obesidade-abeso. Acesso em: 29 out. 2020.

ANSSAR, Tareq $\mathrm{M}$ et al. Autoimmune diseases and immunosuppressive therapy in relation to the risk of glioma. Cancer Med., [s. I.], ano 2017, v. 9, ed. 3, p. 1263-1275, 10 dez. 2019. DOI 10.1002/cam4.2767. Disponível em: https://www.ncbi.nlm.nih.gov/pmc/articles/PMC6997055/. Acesso em: 22 out. 2020.

BENKE, Geza et al. Occupational solvent exposure and risk of glioma in the INTEROCC study. Br J Cancer, [s. I.], ano 2017, v. 117, ed. 8, p. 1246 - 1254, 14 set. 2017. $\mathrm{DOI}$ 10.1038/bjc.2017.285.

Disponível em: https://www.nature.com/articles/bjc2017285. Acesso em: 22 out. 2020. 
BENSON, Victoria S. Menopausal hormone therapy and central nervous system tumor risk: large UK prospective study and meta-analysis. Menopause, [s. I.], 2015. DOI https://onlinelibrary.wiley.com/doi/epdf/10.1002/ijc.29274.

Disponível em: https://pubmed.ncbi.nlm.nih.gov/25335165/. Acesso em: 25 out. 2020.

BERLINER, Matan Ben-Zion et al. Height as a risk factor in meningioma: a study of 2 $\begin{array}{llll}\text { million Israeli } & \text { adolescents. Height, } & \text { s. l.], } & 2020 .\end{array}$ DOI https://bmccancer.biomedcentral.com/articles/10.1186/s12885-020-07292-4.

Disponível em: https://pubmed.ncbi.nlm.nih.gov/32819306/. Acesso em: 25 out. 2020.

BERTOLUCCI, Paulo H. et al. Guias de medicina ambulatorial e hospitalar da unifesp-epm: neurologia. 1. ed. São Paulo: Manole, 2011. ISBN 978-85-204-5239-4.

DISNEY-HOGG, Linden et al. Impact of atopy on risk of glioma: a Mendelian

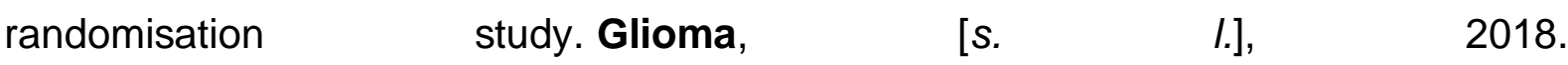
DOI https://bmcmedicine.biomedcentral.com/articles/10.1186/s12916-018-1027-5.

Disponível em: https://pubmed.ncbi.nlm.nih.gov/29540232/. Acesso em: 25 out. 2020.

KATZ, Artur et al. Tratado de Oncologia. São Paulo: Editora Atheneu, 2013. ISBN 978-85-388-0312-6.

MCELVENNY, Damien $\mathrm{M}$ et al. The INTEROCC case-control study: risk of meningioma and occupational exposure to selected combustion products, dusts and other chemical agents. Occup Environ Med , [s. I.], ano 2017, v. 75, ed. 1, p. 12-22, 2017. DOI 10.1136/oemed-2016-104280. Disponível em: https://oem.bmj.com/content/75/1/12. Acesso em: 22 out. 2020.

MENDES, G. A. et al. Epidemiologia de uma série de tumores primários do sistema nervoso central. Porto Alegre, RS, Brasil: Arquivo Brasileiro de Neurocirurgia, p. 279-283; 2014.

MUSKENS, Ivo $S$ et al. Body mass index, comorbidities, and hormonal factors in relation to meningioma in an ethnically diverse population:: the Multiethnic Cohort. Hormonal Factors, [s. I.], 2019. DOI https://academic.oup.com/neuro- 
oncology/article-abstract/21/4/498/5274483?redirectedFrom=fulltext.

Disponível em: https://pubmed.ncbi.nlm.nih.gov/30615143/. Acesso em: 25 out. 2020.

PARENT, Marie-Elise et al. Lifetime occupational exposure to metals and welding fumes, and risk of glioma: a 7-country population-based case-control study. Environ Health , [s. I.], ano 2017, v. 16, n. 90, 25 ago. 2017. DOI 10.1186 / s12940-017-0300y. Disponível em: https://ehjournal.biomedcentral.com/articles/10.1186/s12940-0170300-y. Acesso em: 22 out. 2020.

POLLAK, Michael N. Investigating Metformin for Cancer Prevention and Treatment: The End of the Beginning. American Association for Cancer Research , [S. I.], p. 114, 27 ago. 2012. DOI 10.1158/2159-8290.CD-12-0263. Disponível em: https://cancerdiscovery.aacrjournals.org/content/2/9/778.short. Acesso em: 29 out. 2020 .

POUCHIEU, Camille et al. Allergic conditions and risk of glioma and meningioma in the CERENAT case-control study. Glioma, [s. l.], 2018. DOI https://link.springer.com/article/10.1007/s11060-018-2816-6. Disponível em: https://pubmed.ncbi.nlm.nih.gov/29500663/. Acesso em: 25 out. 2020.

R ROGERS, Lisa et al. Association of metabolic syndrome with glioblastoma: a retrospective cohort study and review. Neuro-Oncology Practice, [s. I.], v. 7, 2020. Disponível em: https://academic.oup.com/nop/articleabstract/7/5/541/5813864?redirectedFrom=fulltext. Acesso em: 15 out. 2020.

SADETZKI, Siegal et al. Occupational exposure to metals and risk of meningioma: a multinational case-control study. J Neurooncol , [s. I.], ano 2016, v. 130, ed. 3, p. 505 - 515, 23 set. 2016. DOI 10.1007/s11060-016-2244-4. Disponível em: https://link.springer.com/article/10.1007/s11060-016-2244-4. Acesso em: 22 out. 2020.

SCHWARTZBAUM, Judith. Associations between prediagnostic blood glucose levels, diabetes, and glioma. Scientific Reports, [S. I.], p. 1-9, 3 maio 2017. DOI DOI:10.1038/s41598-017-01553-2. 
https://www.nature.com/articles/s41598-017-01553-2\#citeas. Acesso em: 29 out. 2020.

SELIGER, Corinna et al. Diabetes, use of antidiabetic drugs, and the risk of glioma. Neuro Oncol., [s. I.], ano 2015, v. 18, ed. 3, p. 340-349, 20 jun. 2015. DOI 10.1093/neuonc/nov100.

https://www.ncbi.nlm.nih.gov/pmc/articles/PMC4767232/. Acesso em: 22 out. 2020.

SELIGER, Corinna et al. Diabetes, use of metformin, and the risk of meningioma. PLoS One, [s. I.], ano 2017, v. 12, ed. 7, 14 jul. 2017. DOI 10.1371 / journal.pone.0181089. https://www.ncbi.nlm.nih.gov/pmc/articles/PMC5510861/. Acesso em: 22 out. 2020.

SELIGER, Corinna et al. Metabolic syndrome in relation to risk of meningioma. Oncotarget, [s. I.], ano 2016, v. 8, ed. 2, p. 2284-2292, 26 nov. 2016. DOI $\quad 10.18632$ / oncotarget.13667. Disponível em: https://www.oncotarget.com/article/13667/text. Acesso em: 22 out. 2020.

TAKAHASHI, Hannah et al. Mendelian randomization provides support for obesity as a risk factor for meningioma. Mendelian randomization, [s. I.], 2019. DOI https://www.nature.com/articles/s41598-018-36186-6.

Disponível em: https://pubmed.ncbi.nlm.nih.gov/30670737/. Acesso em: 25 out. 2020.

TURNER, Michelle $\mathrm{C}$ et al. Interactions between occupational exposure to extremely low frequency magnetic fields and chemicals for brain tumour risk in the INTEROCC study. Occup Environ Med, [s. I.], ano 2017, v. 74, ed. 11, p. 802-809, 2017. DOI 10.1136 oemed-2016-104080.

Disponível

em: https://oem.bmj.com/content/74/11/802.long. Acesso em: 22 out. 2020.

WIEDMANN, Markus $\mathrm{K} \mathrm{H}$ et al. The impact of body mass index and height on the risk for glioblastoma and other glioma subgroups: a large prospective cohort study. Body mass index, [s. l.], 2017. DOI https://academic.oup.com/neurooncology/article/19/7/976/2769495.

Disponível em: https://pubmed.ncbi.nlm.nih.gov/28040713/. Acesso em: 25 out. 2020. 
WIEDMANN, Markus K. H. Overweight, obesity and height as risk factors for meningioma, glioma, pituitary adenoma and nerve sheath tumor: a large populationbased prospective cohort study. Acta Oncologia , [S. I.], p. 1-9, 29 out. 2020. DOI 10.1080/0284186X.2017.1330554.

em:

https://pubmed.ncbi.nlm.nih.gov/28548875/. Acesso em: 29 out. 2020.

WIEDMANN, Markus $\mathrm{KH}$ et al. Overweight, obesity and height as risk factors for meningioma, glioma, pituitary adenoma and nerve sheath tumor: a large populationbased prospective cohort study. Acta Oncologica, [s. I.], ano 2017, v. 56, ed. 10, p. 1302-1309, 26 maio 2017. DOI 10.1080/0284186X.2017.1330554. Disponível em: https://www.tandfonline.com/doi/full/10.1080/0284186X.2017.1330554. Acesso em: 22 out. 2020.

ZHANG , Rio. Body mass index and risk of brain tumors: a systematic review and dose-response meta-analysis. European Journal of Clinical Nutrition, [S. I.], p. 757-765, 24 fev. 2016. DOI https://doi.org/10.1038/ejcn.2016.4. Disponível em: https://pubmed.ncbi.nlm.nih.gov/26908425/. Acesso em: 29 out. 2020.

ZHANG, D. Body mass index and risk of brain tumors: a systematic review and doseresponse meta-analysis. Body mass, [s. l.], 2016. DOI https://www.nature.com/articles/ejcn20164. em: https://pubmed.ncbi.nlm.nih.gov/26908425/. Acesso em: 25 out. 2020.

Enviado: Novembro, 2020

Aprovado: Janeiro, 2021. 